\title{
Guiding Principles for Successful Enterprise Performance Solution System Implementations
}

\author{
Thomas A. Woolman ${ }^{1}$ \\ ${ }^{1}$ On Target Technologies, Inc., Amissville, Virginia, United States \\ Correspondence: Thomas A. Woolman, On Target Technologies, Inc., 91 Battle Mountain Road, Suite 201, \\ Amissville, VA 20106-4341 U.S.A. Tel: 1-215-805-7357. E-mail: twoolman@ontargettek.com
}

Received: July 11, 2014

Accepted: August 14, 2014

Online Published: September 25, 2014

doi:10.5539/ijbm.v9n10p143

URL: http://dx.doi.org/10.5539/ijbm.v9n10p143

\begin{abstract}
Enterprise Performance Solution Systems (EPSS) encompass a critical niche capability within the greater spectrum of Business Analytics systems. Significant financial reporting and management theory challenges relate to the design, implementation and successful utilization of these powerful corporate capital planning and analysis systems. Chief among the technical challenges are the metadata and fact data integrations with legacy, data warehouses and ERP repositories. Management theory challenges may be even more significant and relate to conflicts between and within corporate information technology and financial reporting cultures, data governance practices, and understanding oftentimes disparate forecasting and budgeting methodologies within aspects of the organization. Guiding principles are explored in this paper with the objective of mitigating common technical and functional area hurdles which can impact the success of these implementations. Strategies for enhancing the system design process and interactions between operational management end users and system designers are also explored.
\end{abstract}

Keywords: enterprise performance solution system, enterprise performance management, enterprise capital planning, multidimensional, planning systems, business intelligence, business analytics, business process management, change management optimization, ad hoc reporting, enterprise planning

\section{Background of the Research}

Enterprise Resource Planning (ERP) systems are now ubiquitous (Rashid et al, 2002) within large corporate enterprises and government agencies and are now well within the stage of being repackaged for implementation within smaller to medium enterprises (SMEs). In conjunction with the widespread adoption of data warehousing concepts driving towards a holistic data governance practice, the implementation of Enterprise Performance Solution Systems (EPSS) becomes a logical and necessary outgrowth of government and corporate financial management capabilities. An EPSS, also commonly known as an Enterprise Performance Management (EPM) system, is defined in this paper as a subset of the universe of Business Intelligence (BI) systems. This system is focused on the monitoring, planning, and managing the performance of an enterprise.

With the increasing adoption of EPSS, significant challenges within the organization can arise that impede the successful design, development and adoption of these systems. The major impediment categories can be broken into two segments: organizational management and information management practices. The organizational management challenges and recommended guidelines for mitigation are first addressed in this article, then followed by the information management hurdles and potential remediation solutions.

\section{Methodology}

This EPSS field research was undertaken by using a combination of direct observation or survey-based answers in lieu of direct observation. EPSS field research was targeted at American-based multinational for-profit enterprises in the Global 1000 category. These were further narrowed to include as having recently concluded (successfully for unsuccessfully) or were currently in progress of either concluding or terminating a customized EPSS implementation project. Issues were reported by all 4 surveyed enterprises, as defined below. 
Table 1 . The issues survey reports

\begin{tabular}{|c|c|c|c|c|c|}
\hline & $\begin{array}{l}\text { Financial } \\
\text { Reporting } \\
\text { Fiefdom Issues }\end{array}$ & $\begin{array}{l}\text { Availability of } \\
\text { Organizational } \\
\text { Metadata Across } \\
\text { Enterprise }\end{array}$ & $\begin{array}{l}\text { Structured and } \\
\text { Synchronized } \\
\text { Metadata and } \\
\text { Legacy Fact Data }\end{array}$ & $\begin{array}{l}\text { CFO-Level } \\
\text { Engagement }\end{array}$ & $\begin{array}{l}\text { EPSS Implementation } \\
\text { Success Rating }\end{array}$ \\
\hline $\begin{array}{l}\text { Company } \\
\text { "A" }\end{array}$ & No & Yes & Yes & Yes & Significant Success \\
\hline $\begin{array}{l}\text { Company } \\
\text { " } B \text { " }\end{array}$ & Yes & Yes & $\begin{array}{l}\text { Available but } \\
\text { issues ongoing }\end{array}$ & Moderate & $\begin{array}{l}\text { Successful, but under } \\
\text { continuous } \\
\text { improvement }\end{array}$ \\
\hline $\begin{array}{l}\text { Company } \\
\text { "C" }\end{array}$ & Yes & No & No & Negligible & Failure \\
\hline $\begin{array}{l}\text { Company } \\
\text { " } D \text { " }\end{array}$ & No & Yes & $\begin{array}{l}\text { Availablebut } \\
\text { issues ongoing }\end{array}$ & Yes & $\begin{array}{l}\text { Successful, but limited } \\
\text { in scope }\end{array}$ \\
\hline
\end{tabular}

Results of this survey data are discussed in the conclusions section, after next incorporating key concepts related to each assessment category.

\section{Organizational Management Barriers to Success}

\subsection{Financial Reporting Fiefdoms}

Financial reporting fiefdoms, in which disparate divisions within an enterprise are unwilling or unable to adopt enterprise financial planning standards will quickly lead the EPSS initiative into a roadblock that is not easily recovered from. Separate divisions which undertake their own internal hedging strategies, retain internal and uncoordinated financial reporting system teams, and justify their current procedures by being focused more on their local division-centric management reporting rather than corporate financial standards retain a key stake in maintaining the status quo and avoiding the adoption of integrated enterprise standard systems.

Key stakeholders within these various financial fiefdoms need to be incentivized to recognize the inherent return on investment and organization synergies intrinsic with the successful design, implementation and adoption of the new EPSS. By integrating the fiefdoms through a motivational catalyst into success of the EPSS and the corresponding financial planning and reporting solution standards, key personnel will sense an ownership stake in the adoption and thriving acceptance of the new system and reduce their barriers to adoption.

Discussions of several (Herbold, 2004) of the most harmful types are the enterprise fiefdoms, which often involves a relatively small team centered on objectives, common tasks or shared responsibilities must be undertaken. Common examples of this group fiefdom include divisional finance teams within an enterprise subsidiary. Similar to the divisional-driven or geographic-centered fiefdom but more harmful is the group fiefdom, which is often centered on some set of tasks related to a segment of corporate finance. The finance team at Enron is a classic example of a group fiefdom, in that it self-servingly filtered information for immediate gain without adequately advising senior corporate executives.

The less insidious type, the divisional fiefdom, almost always arise within larger enterprises that have multiple lines of business and operations. These fiefdoms then drive behaviors around a single range closely-bound of entities. Without the necessary synergy with the parent corporate division, it disregards the operational objectives related to the greater good of the organization. This fiefdom functions solely from the viewpoint of serving the self-interest either directly or implicitly of the local division and will not accept any change management standards of their own accord.

These fiefdoms exist largely out of a natural human desire to control information about them and their immediate area of operations, in an effort to manage appearances. Incentives (Chen, 2011) however need to be in place to promote the existence of a transparent and performance driven meritocracy within the enterprise. The first step in enabling that process is by removing the controlling barriers to information and process management, and ultimately change management.

This enables different aspects of the enterprise to be comparatively analyzed with standardized metrics impartially. Perhaps even more useful is the secondary ability that then allows these divisions to be rated against external competing businesses or agencies within comparable fields of expertise. The end result of the elimination of management fiefdoms is the empowerment of key sponsors who are funding the EPSS initiative to drive the success of the overall enterprise. 


\subsection{Organizational Metadata Structure Synergy}

It might seem obvious on the surface, but in practice it is both uncommon and critical for an enterprise to standardize the definition and use of key business metadata terms related to structural hierarchies. Having a common metadata dictionary that precisely defines what is the definition of such terms as an entity, a region, or a business area is mandatory. Likewise, understanding their current disjointed, incoherent applications across the different divisions of the organization is also key. This enables divisions to communicate with the enterprise and the later to have a functional awareness of challenges faced by the division. This will lead to the understanding of the different and possibly conflicting information process flows within the current state of the enterprise that need attention and can be addressed by the EPSS.

Without addressing and resolving these terminology standards first, confusion will reign supreme with the implementation and attempted adoption of the EPSS. Confusion will in term prevent acceptance of the truth contained within the new EPSS by organizational finance and senior management, who may be culturally acclimated to a distinct functional viewpoint and unaware of divisional and entity-specific terminology. The worst case scenario could be adoption of the system but disuse, leading to disharmony in financial capital planning processes throughout the organization and no return on investment.

\subsection{Divisional Overhead Reduction Is a Driving Goal}

The automation and optimization of the enterprise capital planning system leads to an inevitable stakeholder return on investment opportunity that is largely driven by staffing realignment. Recognizing the overhead burden within organizational planning layers are fundamentally redundant across divisional boundaries, a change management process would ideally by synchronized after the implementation of the EPSS. This realignment opportunity could direct some resources to revenue generating areas of the enterprise for example, which the EPSS itself might have identified via use of labor and revenue KPIs. It was discussed (Presley et al, 1997) that collaboration under the guidance of a participative development approach to drive the development and quantification of these performance metrics is advisable. This was in order to accurately present to the key stakeholders a reflection of the capital structure of the enterprise via the EPSS.

By integrating this collaborative development approach including reviews of the new system metrics, it supports the value and acceptance of the new system. Further, the fiefdoms described above are almost universally aware of the opportunities that the EPSS presents to their internal customers (the stakeholders). Their natural posture would be skepticism with regard to the acceptance and success of the system as it effects their division, therefore senior management must carefully monitor and intervene in an active, impartial manner before acceptance risk or obstructions materialize.

\subsection{Non-Enterprise Standard Legacy Transactional Systems Should Be Reduced and Ultimately Replaced}

In many respects the preservation of legacy strategic information systems which predate the EPSS initiative and have discordant metadata structures and fact data granularities and application principles are tied to the aforementioned fiefdoms. An example of this might be a sales forecasting system utilized by a single segment of the enterprise, most likely a previous external acquisition. The temptation by senior management might be to leave much of said division's corporate culture and legacy systems intact, with the belief that this is part of the asset base that was purposely acquired and it provides net positive return on investment to the enterprise.

While this may have initially been the case during the immediate post-acquisition phase, a failure or reluctance to integrate the mature dissonant legacy systems can cause havoc with layering an EPSS or other BI analytical system on top. Bringing these legacy systems to an end of life migration and harmonization of metadata and fact data standards with the enterprise prevailing applications before undertaking the strategic capital systems initiative is critical to long term success.

\subsection{The CFO must Be Actively Involved}

Due to the cross-divisional nature of EPSS implementations, the administrative and data governance dynamics will leave an insurmountable number of roadblocks in front of the implementation management team without sufficient enterprise governance support. Conflicting or unclear mission statements and instructions related to financial metrics desired and process management changes desired will negate the value of senior management involvement.

Likewise, active senior management involvement can preempt fiefdom issues, align internal finance resources for validation and process flow mappings, and promote timely acceptance procedures are followed. This is not to say that the CFO would replace the normal project management senior staffing role, but the involvement beyond the initial sponsorship initiation is required at this strategic junction is required in an ongoing basis in a liaison 
role with the senior project management. Without direct CFO sponsorship and involvement, business process standardization which drives the EPSS functions cannot take place and success of the system becomes in question.

\section{Enterprise Information Systems Barriers to Success}

As is the case with the aforementioned business process management barriers, enterprise information system hurdles can pose an equally daunting challenge to the successful EPSS implementation. In many ways these are intimately tied to the business process management hurdles and can be proactively addressed while still in the system planning and design stages so that solutions can be addressed before implementation begins.

\subsection{Managing Multiple Synchronous Data Feeds}

EPSS systems typically retrieve information from multiple sources (Januszewski, 2009) simultaneously in order to provide a holistic view of the consolidated functions within the enterprise. This data is oftentimes at both different levels of granularity (contract line items versus projects, for example) and may even have different dimensionality constraints. Integration solutions must be defined to address these issues within the framework of an ETL (extract, transform, and load) solution.

The ETL will likely need to be custom built as part of the EPSS project and can encompass a significant amount of work depending on the number of applications within the EPSS system and the number of transactional source systems that will feed into it. Giving sufficient time and resources to both accurately assess the scope and breadth of this ETL undertaking as well as testing the accuracy and transformed dimensionality of the post-processed transactional data into the EPSS should be stressed. Data validation and systems integration testing, including batch automation processes, should be scheduled well in advance. This component of the system should also be treated to a significant degree as a stand-alone component; thereby its unit testing, data validation and systems integration and automation testing can be conducted in parallel while the core EPSS system development continues to take place.

\subsection{Conference Room Pilot and Senior Management Sign-Off}

The implementation phase must have a pilot demonstration component which integrates all or at least portions of the ETL data load process and metadata feeds. The pilot will serve as a validation of requirements gathering, consolidation and calculation methodologies and the end user experience. While it would not normally be expected that all data views and all metrics would be fully functional, sufficient functionality should be demonstrable so that senior management can review, critique and then officially sign off on the pilot. Pilot sessions would typically be scheduled for each application within the EPSS, early enough in the implementation phase so that issues noted by senior management can effectively be mitigated.

Likewise, aspects of the user experience such as specific web page functionality may not yet fully be integrated or available, but enough capability should be present so that gaps are fully documented and the expected performance is understood by senior management. Provided the conference room pilot can be a bidirectional process however.

In such a process, functional users and senior management can collaborate during these sessions to both better understand what is to be delivered, and provide an opportunity to make adjustments while development is still in flight. These conversations should be encouraged and not be considered an unexpected situation. In many development instances, particularly when an EPSS which has not existed before, operational and senior management end users will be learning about their needs relative to the system's capabilities and assumptions as well as the business needs analysis will be continuously evolving. In situations where significant change management is being introduced from the conference room pilot, a waterfall process may need to be initiated. In such a process, short duration revision-review-approval mini pilot follow-ups will occur to resolve issues. Documentation and sign-off on each of these review sessions is critical to keep the project on focus and on track.

\subsection{Metadata Management Solution Strategies}

In conjunction with integrating multiple synchronous fact data sources through the use of an ETL framework, the perquisite for transactional data feed governance is having stable metadata feeds driven by these same sources. While some EPSS technologies are more forgiving of "dirty" metadata than others, having a properly synchronized and agreed upon chart of accounts, consistent definitions of dimensionality concepts, clearly defined international financial entities, and departmental and human resource areas defined and consistent will make the system integration, development and testing far more efficient. All of these metadata feeds must be centrally managed with the adoption of a Master Metadata Management solution. 
Without these standards in place before fact data ETL processes are started, it will make data validation and system testing far more difficult and will risk slowing the progress of the project dramatically. In a worst case scenario, it may even slow down or derail entirely the conference room pilots.

\subsection{Formalized Reporting vs Planning Queries}

The enterprise must understand the differentiation between formalized reporting and ad-hoc queries supporting the enterprise capital planning process. In particular, senior management and stakeholders must understand the different audience for each of these capabilities, including the need for on-demand ad hoc query analytics. With a Formalized Reporting Strategy, structured financial information is provided in a pre-defined specific layout and is intended for internal or even external applications. Metadata and fact data security are always a part of the Formalized Reporting Strategy therefore many reports themselves will be automated to execute under an automated system account. While some categories of users can be empowered to define their own management report criteria, even these reports would remain in the formal category due to the pre-defined nature and intended appearance of these queries.

Planning ad-hoc queries on the other hard are structures that are intended to support specific tactical aspects of the overall enterprise planning process including labor, expense, revenue, balance sheet, cash flow and income statement components. In many cases these separate components many actually be broken into individual applications within the overall system in an effort to better manage different granularities and dimensionality needs. Each of these components within the planning system can be further subdivided into (for example, but not limited to) budget (AOP) and forecast, as well as a variety of what-if scenarios available to support various cases of interest to senior management stakeholders. These various tactical level needs can be managed by a series of user interfaces that are driven by security rights, allowing a combination of read and write pre-defined queries as well as the capability to create on-demand informal ad-hoc queries that are governed by security authorizations.

\subsection{The Role of Enterprise Architecture Integration}

The role of integrating the EPSS into the overarching enterprise system architecture plan should not be minimized. Because the nature of these systems is largely oriented to corporate finance and senior management within the organization, it is tempting to think of the EPSS as separate from the information technology segment of the business. Oftentimes a portion of the technologies and vendor tools utilized by the EPSS will be unique to other platforms managed and automated by the information technology section, but that should not be sufficient reason for IT to not plan on formally supporting the installation and maintenance. While corporate finance, typically under the leadership of a Vice President of Enterprise Financial Planning and Analysis will have responsibility for developing and validating and usage of the EPSS, enterprise information technology plays a critical role in properly keeping the system operational. Assuring data accessibility from legacy source systems, backup and recovery processes, virtual server cluster management, integrations with metadata management tools and potentially multiple distributed relational database schemas utilized by the EPSS are among the many aspects of corporate IT support roles.

\section{Conclusion}

From the EPSS field research, a clear pattern emerged within the above field research survey data. Key amongst the response criteria relative to the EPSS Implementation Success Rating were the availability and overall condition of metadata and synchronized fact data across the enterprise. CFO level engagement clearly facilitated success factors as did the presence of financial reporting fiefdoms. But without the metadata and fact data synchronization and access issues resolved beforehand, the success of an EPSS implementation may likely be hampered by fragmented, ongoing mitigation issues.

The benefits of complete implementations of enterprise (also referred to as strategic) performance management systemsincluded (Wall \& Nijkamp, 2009) both quantitative and qualitative advantages. Quantitative advantages included a visible net impact on revenues, net profits and gross sales. Shortcomings in legacy EPSS systems included one-dimensional financial information and a lack of focus on competitive advantages that drove the business process, as well as lacking in robust and timely strategic intelligence data.

System reengineering enhancements to legacy EPSS or complete implementations of new initiatives have often included recognizing the non-financial measures for benchmarking achievements within the enterprise (Malina $\&$ Selto, 2001). These implementations often resulted in increased revenue and profits as a quantitative advantage, and in many instances improvements in the corporate collaborative environment and organizational synergiesfollowed qualitatively (Lovell et al., 2002). 
This paper analyzes some of the commonly encountered corporate management and enterprise information system challenges encountered with large scale EPSS design and implementations. The intention with this paper is to provide a roadmap that benefits EPSS project management and system architects to make them more successful in their methodologies. The challenges of developing these systems successfully can be many, but the benefits associated with a successful EPSS can vastly outweigh the risks. The benefits to the enterprise and its stakeholders can include increased profitability, reduced costs and greatly reduced management planning cycles.

\section{References}

Chen, D. (2011). Research on Performance Management of Chinese SME. International Journal of Business and Management, 6(4), 265.

Herbold \& Robert, J. (2004). Overcoming the Fiefdom Syndrome: Conquering Office Politics. Business Finance.

Januszewski, A. (2009). Overview of the Business Performance Solutions, Polish Association for Knowledge Management. Series: Studies and Proceedings, 20, 45.

Lovell, B., Radnor, Z., \& Henderson, J. (2002). A pragmatic assessment of the balanced scorecard: an evaluation of a new performance system for use in a NHS multi agency setting in the UK. Working Paper Series, 2(13), 339-346.

Malina, M. A., \& Selto, F. H. (2001). Communicating and controlling strategy: an empirical study of the effectiveness of the balanced scorecard. Journal of Management Accounting Research, 13, 47-90.

Presley, A. R., Whitman, L. E., \& Donald, H. L. (1997). A methodology for enterprise performance management. Proceedings of the 1997 Decision Sciences Institute Annual Meeting. San Diego, CA. Wiley-Blackwell.

Rashid, M. A., Hossain, L., \& Patrick, J. D. (2002). The evolution of ERP systems: A historical perspective. Enterprise Resource Planning: Global opportunities \& challenges, 1-16.

Waal, A., \& Nijkamp, K. (2009). The relationship between the level of completeness of a strategic performance management system and perceived advantages anddisadvantages. International Journal of Operations \& Production Management, 29(12), 1242-1265.

\section{Copyrights}

Copyright for this article is retained by the author(s), with first publication rights granted to the journal.

This is an open-access article distributed under the terms and conditions of the Creative Commons Attribution license (http://creativecommons.org/licenses/by/3.0/). 